\title{
Research on Building Technology of Aquaculture Water Quality Real-Time Monitoring Software Platform
}

\author{
Yinchi Ma ${ }^{1,2}$, Wen Ding ${ }^{1,2}$, Wentong $\mathrm{Li}^{1,2}$ \\ 1 Beijing Fisheries Research Institute, Beijing, 100068, China \\ 2 National Engineering Research Center of Freshwater Fisheries, Beijing, 100068, China
}

\begin{abstract}
At present, the information level of the aquaculture water quality monitoring is relatively backward in China. Building a digital, networked, intelligent real-time dynamic aquaculture water quality monitoring system by the modern electronic information technology, communications technology and wireless sensor network technology will have an important significance for the factory aquaculture technological innovation. The system is not only able to detect the main indicators of the aquaculture water quality (temperature, $\mathrm{PH}$, dissolved oxygen, turbidity, ammonia, etc.) in real-time, but also be able to do the data fusion and data mining through its software platform, and establish the water quality indicators historical database. This software platform can achieve display and analysis of the local or remote monitoring data in real-time and dynamically, which can provide an important technical means and scientific basis to improve the water utilization effectively, and reduce the pollutant emission in aquaculture. This article will focus on the building technology of the system software platform.
\end{abstract}

Key words: aquarium; wireless sensor network; software platform; monitoring

\section{Introduction}

Wireless sensor network is a wireless network without infrastructure, which combines sensor technology, embedded computing technology, modern network technology, wireless communication technology and distributed intelligent information processing technology. It can work unattended during a long time ${ }^{[1]}$. In this study, the wireless sensor network technology is used to build an intelligent, network-based aquaculture water quality wireless monitoring system. In addition, we build a water quality monitoring data intelligence analysis software platform used $\mathrm{VC}++$ program language and database technology, which provides an information and visualization interface for the aquaculture technicians. The implementation of this system can fundamentally enhance the aquaculture water quality monitoring level, which is relatively backward ${ }^{[2] \sim[5]}$. This paper will focus on the design and implementation of data storage module, analysis module and display module within the software platform.

\footnotetext{
${ }^{1}$ Ma Yinchi (1982 -), Beijing Fisheries Research Institute, Senior Engineer, Master, graduated from Beijing Normal University, State Key Laboratory of Remote Sensing Science, mainly engaged in research of agriculture remote sensing and fisheries information technology.
} 


\section{Function and Feature of Software Platform}

The System transmits the monitoring data to the monitoring center through the wireless communication technology. It can realize the continuous data recording, analysis, processing and warning in real-time, so that it can remind the aquaculture technician to take the necessary adjustment measures at first time. This Method can effectively guarantee the aquaculture safety under intensive farming condition. The system software platform can realize the following functions:

I. The system can monitor the various water quality parameters automatically during 24-hours in a whole day, and the data is sent to the center server automatically, while the online records and related analysis will be done.

II. Data server will collect each node data, authorized customers can remote login the data server to inquiry the experimental data or have aided analysis at any time through PC installed the client software.

III. The data analysis system can display monitoring data of each sampling node through graphical and tabular form, which provides user with an intuitive water quality trends. At the same time, the collected data will be saved, which will be added to the large-capacity water quality history database. And this history database can provide a scientific basis for production.

The structure design of the system software platform is shown as follow (Fig.1).

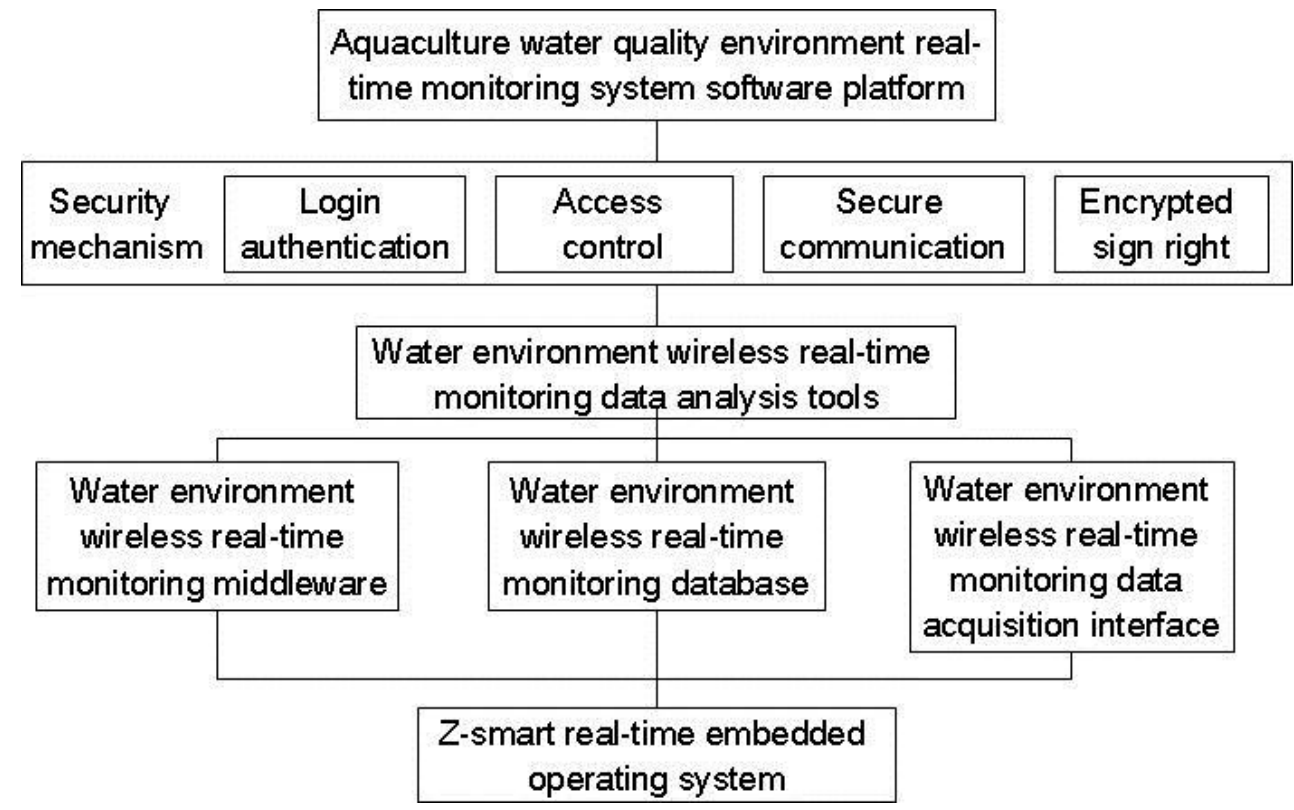

Fig. 1. Structure Design of System Software Platform

The main features of the system software platform include:

I. Remote real-time and intelligent monitoring.

The system combined with advanced embedded computing technology, modern networking and wireless communications technology to automatically collect various sensing parameters to achieve a real-time and intelligent remote monitoring of water quality.

II. Professional and friendly interface.

The software platform is developed for the application of aquaculture water quality. User can easily view the real-time water quality condition of each aquaculture pond through the 
software platform interface, as well as the software platform also has a variety of chart and graph display. The Rich display forms of data enhance the usefulness of this system.

III. Powerful database system.

Using the distributed database technology, the platform realizes a multi-functional, highly integrated data service system for the water quality monitoring, which integrated data analysis, data management and data integration display. In addition, the software platform also has a strong storage and query capability for the history data, and it can provide user with comprehensive and reliable data service.

\section{Design and Development of Software Platform}

The real-time monitoring software platform of aquaculture water quality is self-developed upper application for the water quality monitoring with independent intellectual property rights. The platform is designed for the special needs of the aquaculture industry. User can monitor the aquaculture water environmental parameters such as water temperature, $\mathrm{PH}$, dissolved oxygen, turbidity, ammonia and other data by the on-line monitoring platform, which makes advance warning of the unknown water quality mutation, and effectively curb the risk of aquaculture and significantly improve production and management efficiency. It uses the hierarchical structure technology, which can be adjusted as needed, and dynamically changes the kernel size. In addition, as the software abstract of system hardware, it is primarily responsible for managing and driving the underlying hardware, optimizing network structure, timely detection, controlling the flow of network traffic, ensuring the quality of network application services and extending service hours. In this paper, the network layer protocol stack and middleware technology has been developed based on Z-smart and provided the high-quality application software product for the information processing and service needs of the variety water quality testing applications.

The middleware development is one of focal points of the system. The technology will greatly shorten the development period of the application System.

\subsection{Design and Development of Underlying Platform}

The real-time embedded operating system called Z-smart by independent research and development is designed for wireless sensor. The middleware is a standard intermediate layer structure with a common property. It is responsible for sensor network system development support for the resource management, dynamic environment analysis and ubiquitous application, such as application change, extension, system upgrades, reused and the other key properties. A key function of the middleware is conversion and agent of heterogeneous networks, such as explaining the data format of sensor network correctly and converting it into TCP / IP data or reverse operation. Therefore, the middleware must have rapid processing capability.

\subsection{Design and Development of Application Layer Function Module}

For user, the core of wireless sensor network is the perception data instead of the network hardware. User is interested in the data generated by the sensor, instead of the sensor itself. The 
basic idea of data-centric wireless sensor networks is that the sensor is regarded as the perception of data stream or data source, and the wireless sensor network is regarded as the sensing data space or sensing database. And the data management and processing is regarded as an application target of the network. Based on this idea, the platform application server modules include data loader, data analysis (application analysis) and man-machine interface.

I. Data loader.

The software platform changes data with sensor data collection terminals and data relay node by wireless data interface. In the terminal embedded software development, the system follows the IEEE802.15.4 standard, and software platform communication module is in accordance with the Zigbee Alliance international standards. The complete Zigbee protocol suite is consists of high-level application specification, the application convergence layer, network layer, data link layer and physical layer. The layers apart from the network layer protocol are developed by the Zigbee Alliance, and IEEE Association is responsible for the physical layer and link layer standards.

II. Data analysis.

The system uses data mining technology. User can obtain the needed information from vast amounts of data. In addition, the system also realizes the mining in real-time and mining after the event of the data stream. Data mining refers information processing for decision-making and assessment, in which the large amount of data is automatically analyze and synthesize under certain criteria. In the high-capacity wireless sensor network, a lot of sensing data will be generated in real-time. On the one hand some data is failure data, on the other hand valid data need to be fused together to produce meaningful information. In this case, data mining techniques need to be used. The data is judged and integrated to reduce unnecessary loss of bandwidth and reduce the overall energy consumption so as to provide more meaningful data.

Data mining combines the experience of early information fusion in the commercial applications and military field. According to the center distributed and decentralized distributed judgment ruling theory, tree fusion estimation algorithm is used to realize comprehensive intelligent decision of large amounts of data. The data mining technique of wireless sensor network emphasizes the three core areas of the information integration: First, the information fusion is to complete the process of multi-source information on several levels, where each level representing information abstraction with a different level. Second, information fusion including detection, interconnect, relevant, estimates and information portfolio. Third, the results of the information fusion include the status or identity estimation on the lower level, and the overall decision-making on the higher level.

The system software platform uses multi-sensor data mining technology. And this technology can reduce interference of the invalid data, reduce information redundancy, and improve accuracy. It provides an effective means for the purpose of large amounts of data analysis on the complex system.

III. Man-machine interface.

a. User rights management.

By setting permissions for different users, user can inquiries whether the user is a privilege to observe, manage user permissions, data export privileges, Node Manager or other privileges.

b. Query and graphical display of real-time monitoring point data.

By the list or graphical display of the real-time data, users can view the real-time data of all 
sensor nodes.

c. Graphic display of node deployment.

Display the location of the node deployment and operation status in monitor area visually.

$d$. The treelike display of the monitoring points list.

Users can quickly view the real-time data of each node, node status and other information through this feature.

e. Graphic display of wireless sensor monitoring network topology.

Topology displays data transmission path information between the gateway, relay and the sensor node, as shown in Figure 2.

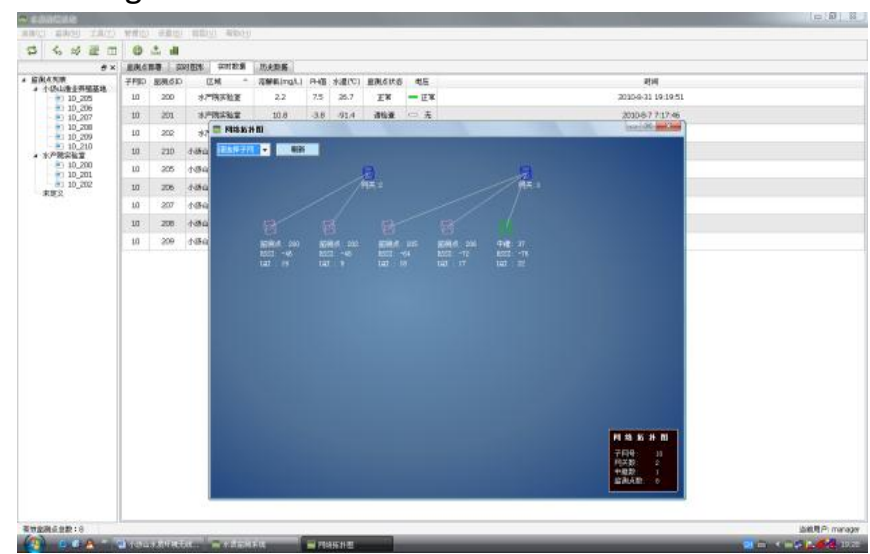

Fig. 2. Display of Topology Diagram

f. Real-time alarm.

Users can set up their own node parameters threshold. When the real-time data of the node exceeds the threshold range, the background color of the node type will be displayed in red, while the node identifier on the node deployment is also displayed in red and the alarm sounds.

g. Query and graphic display of history data.

Historical data can be found all or a specific time period of corresponding node. According to the node number, the sensor type or time period, etc., graphical display can obtain data and showing visual graph, as shown in Figure 3.

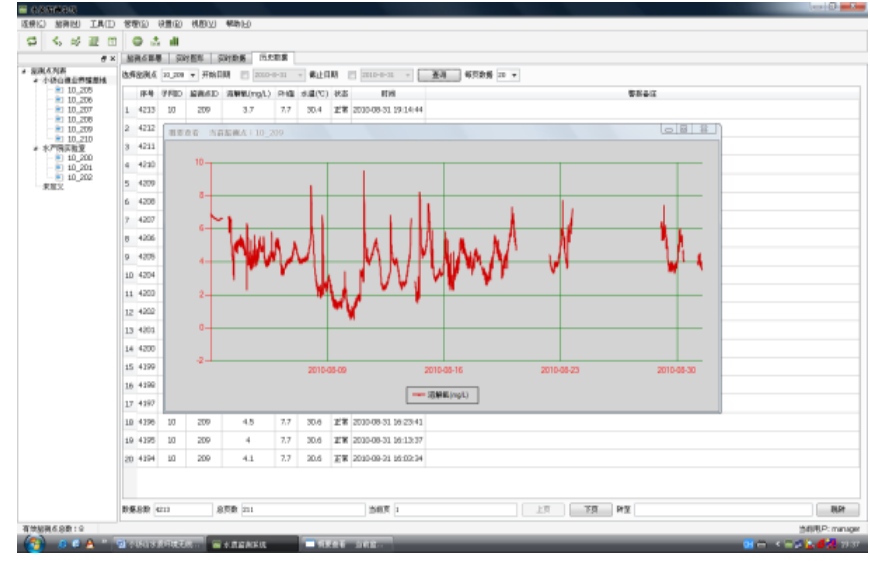

Fig. 3. Graphic Display of History Data

h. Node parameters setting.

Node parameters can be adjusted by users to set send periodic, alias threshold, a coefficient 
of each node, which increases the accuracy level of an individual data collection node.

\section{Conclusions}

This study using wireless sensor network technology to build an intelligent, network-based aquaculture water quality monitoring system, and this practical application of technology in the field of aquaculture promotes the development and application of wireless sensor network technology level powerfully. We propose Zigbee protocol a aquaculture water quality monitoring system software platform solution based on the wireless sensor network technology, and deal with the real-time water quality monitoring data management and analysis.

The results show that based on the software platform, aquaculture technician can obtain a variety of aquaculture water quality indicators, such as water temperature, dissolved oxygen, $\mathrm{PH}$, and turbidity to realize the real-time automatic remote monitoring, wireless data transmission, data processing and analysis, intelligent warning, SMS alarm functions. Thereby this technology can maximize the utilization of aquaculture water to protect the suitable environment conditions for aquaculture animal.

\section{References}

1. Wu Jingtao, Ma Changbao, Liu Yongbo. Design of Wireless Sensor Network Node for Water Quality Monitoring [J]. Computer Measurement \& Control. 2009, 17(12):2575-2578. In Chinese with English abstract.

2. Du Zhi-guo,Xiao De-qin,Zhou Yun-hua,Ou Yang Guo-zhen. Design of water quality monitoring wireless sensor network system based on wireless sensor [J]. Computer Engineering and Design. 2008 29(17):4568-4570. In Chinese with English abstract.

3. Liu Xingguo, Liu Zhaopu, Wang Pengxiang, Miao Lei. Aquaculture security guarantee system based on water quality monitoring and its application [J]. Transactions of the Chinese Society of Agricultural Engineering. 2009, 25(6):186-191. In Chinese with English abstract.

4. Ma Cong-guo,Ni Wei. The design of a factory aquiculture monitor system based on PLC [J]. Industrial Instrumentation \& Automation. 2002, (2):51-53. In Chinese with English abstract.

5. Zhu Wenjin, Ran Gangjun. Research of Agriculture environment Automatic Monitoring and Control [J]. Freshwater Fisheries. 2001, 31(1):60-63. Chinese reverse to English. 